\title{
Early access to bedaquiline for extensively drug-resistant (XDR) and pre-XDR tuberculosis
}

\author{
To the Editor:
}

Globally in 2016, 19\% of previously treated tuberculosis (TB) cases and $4.1 \%$ of newly diagnosed cases were reported to be resistant to isoniazid and rifampicin (multidrug-resistant (MDR)-TB) or rifampicin alone (rifampicin-resistant TB) [1]. In 2017, 8.5\% of MDR-TB cases were caused by extensively drugresistant (XDR)-TB [1], defined as MDR-TB with additional resistance to a fluoroquinolone (FQ) and a second-line injectable drug (SLI) (amikacin, kanamycin or capreomycin). TB drug resistance, especially $\mathrm{XDR}-\mathrm{TB}$, is associated with poorer treatment outcomes [1-3].

Based on phase 2 clinical data, bedaquiline (TMC207), a diarylquinoline antimycobacterial, was granted accelerated or conditional approval in various countries for MDR-TB [4, 5]. Compassionate-use and expanded-access programmes have made bedaquiline available in 95 countries worldwide [6, 7]. "Real-world" data have shown bedaquiline achieves high culture conversion and treatment success rates in patients with MDR-TB and XDR-TB, with a low proportion of adverse events (AEs) [8-13]. In a large-scale study, all-cause mortality was lower in patients treated with bedaquiline $(12.6 \%)$ than in those who received alternative treatments $(24.8 \%)$ [14].

The primary objective of study TMC207TBC3001 (www.clinicaltrials.gov identifier number NCT01464762) was to provide early access to bedaquiline for adult patients ( $\geqslant 18$ years of age) with confirmed sputum smear- or culture-positive TB and drug susceptibility testing results, performed locally as per the local standard of care, demonstrating pulmonary pre-XDR-TB (MDR-TB with additional FQ or SLI resistance) or XDR-TB. The secondary objective was to evaluate safety, tolerability and microbiological status. Enrolled patients had limited to no treatment options and were unable/ineligible to participate in any other bedaquiline study. Results of the 120 -week final analysis are presented here.

Patients received bedaquiline for 24 weeks ( $400 \mathrm{mg}$ orally once daily for 2 weeks and $200 \mathrm{mg}$ orally three times weekly for 22 weeks) with an investigator-selected background regimen (BR) in accordance with national TB programme guidelines. The BR was chosen using three or more drugs to which the TB isolate was known (based on drug-susceptibility testing from within the previous 6 months) or likely (based on known treatment history) to be susceptible, and administered under supervision using the directly observed treatment short-course for MDR-TB (DOTS-Plus). After completing the 24-week bedaquiline plus BR treatment, participants continued to receive BR only for $\leqslant 96$ weeks. Clinic visits were at $2,4,12$, 24, 28, 48, 72, 96 and 120 weeks. Safety, tolerability and microbiological status were assessed throughout. Patients who prematurely withdrew from the study were rigorously followed up to verify survival, unless consent was withdrawn.

The study protocol was reviewed by an independent ethics committee or institutional review board. The study was conducted in accordance with the principles of the Declaration of Helsinki and Good Clinical Practice. All patients provided written informed consent.

Sample size calculation was not performed and no statistical hypotheses were tested. Unless stated otherwise, safety and efficacy analyses were performed on the intent-to-treat (ITT) population, which included all patients who had one or more intake of bedaquiline, regardless of their protocol compliance.

@ERSpublications

This letter presents final safety and efficacy data from an early-access study of bedaquiline for treating (pre-)XDR-TB http://bit.ly/2Ff4qyl

Cite this article as: Vasilyeva I, Mariandyshev A, Kazennyy B, et al. Early access to bedaquiline for extensively drug-resistant (XDR) and pre-XDR tuberculosis. Eur Respir J 2019; 54: 1802208 [https://doi. org/10.1183/13993003.02208-2018]. 
TABLE 1 Patient characteristics, disposition, and safety and efficacy analysis outcomes during 120 weeks

Patient characteristics

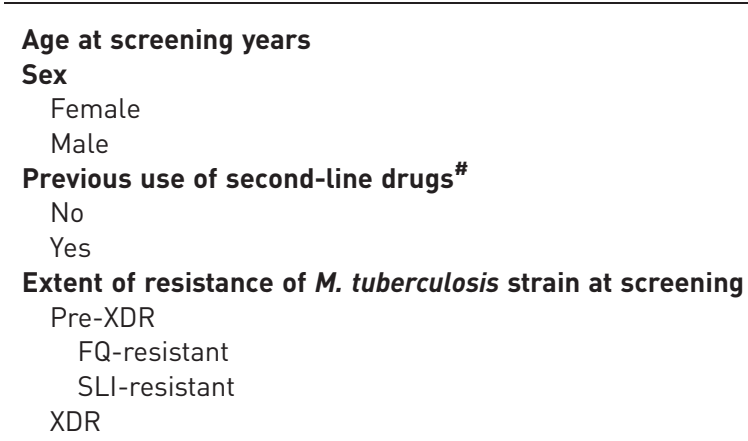

\section{ITT population ( $\mathrm{N}=57)$}

$$
\begin{gathered}
28.0(18-61) \\
33(57.9 \%) \\
24(42.1 \%) \\
4(7.0 \%) \\
53(93.0 \%) \\
27(47.4 \%) \\
14(24.6 \%) \\
13(22.8 \%) \\
30(52.6 \%)
\end{gathered}
$$

\begin{tabular}{|c|c|c|c|}
\hline Deaths & Case 1 & Case 2 & Case 3 \\
\hline Infection subtype & Pre-XDR-TB (FQ resistant) & Pre-XDR-TB (SLI resistant) & XDR-TB \\
\hline Considered related to bedaquiline or BR & No & No & No \\
\hline QTcF at week $24 \mathrm{~ms}$ & 414 & 436 & 310 \\
\hline Sputum culture conversion & No & No & Yes \\
\hline Treatment phase & Post-investigational $^{+}$ & Post-investigational $^{+}$ & Follow-up \\
\hline
\end{tabular}

Resistance to other TB therapies at baseline

$\mathrm{n}$ with observation/ $\mathrm{N}$ tested

\begin{tabular}{lc}
\hline Cycloserine & $3 / 27$ \\
Ethambutol & $44 / 55$ \\
Ethionamide & $31 / 41$ \\
Linezolid & $0 / 5$ \\
PAS & $4 / 30$ \\
Prothionamide & $1 / 2$ \\
Pyrazinamide & $20 / 22$
\end{tabular}

\section{Discontinuations}

ITT population ( $\mathrm{N}=57)$

\begin{tabular}{lr}
\hline Total discontinuations & $14(24.6 \%)$ \\
Discontinuations due to AEs & $5(8.8 \%)$ \\
Deaths & $3(5.3 \%)$ \\
Discontinued bedaquiline & $2{ }^{\text {In }}(3.5 \%)$ \\
Lost to follow-up & $2(3.5 \%)$ \\
Noncompliance & $1(1.8 \%)$ \\
Withdrawal of consent & $2(3.5 \%)$ \\
Patient moved city/country & $4(7.0 \%)$ \\
\hline
\end{tabular}

Safety analysis

Treatment duration weeks

Any $A E$ regardless of cause or severity

Most common ( $>15 \%$ ) AEs regardless of cause or severity

$$
\begin{aligned}
& \text { AST increased } \\
& \text { ALT increased } \\
& \text { Eosinophilia }
\end{aligned}
$$

Any $A E$ leading to discontinuation of bedaquiline

Any $\mathrm{AE}$ at least possibly related to bedaquiline

Most common AEs (>2\%) at least possibly related to bedaquiline $\mathrm{q}^{\text {ศी }}$

AST increased

ALT increased

ECG QT interval prolonged

Any serious $\mathrm{AE}^{++}$

Any grade 3 or $4 A E^{\S \S}$

Most common $(>5 \%)$ grade 3 or $4 \mathrm{AEs}$

$$
\begin{gathered}
97.7(2.1-131.1) \\
50(87.7 \%) \\
28(49.1 \%) \\
15(26.3 \%) \\
13^{\# \#}(22.8 \%) \\
2^{\# \#}(3.5 \%) \\
6(10.5 \%) \\
4(7.0 \%) \\
2(3.5 \%) \\
2(3.5 \%) \\
8^{\# \#}(14.0 \%) \\
15^{\# \#}(26.3 \%)
\end{gathered}
$$


TABLE 1 Continued

Safety analysis

ALT increased, grade 3

AST increased, grade 3

$5^{\# \#}(8.8 \%)$

$4^{\# \#}(7.0 \%)$
Sputum culture conversion over time

Week 24

Week 72

Week 120

Last visit $\mathrm{n}$ with observation/ $\mathrm{N}$ tested

$31 / 36(86.1 \%)$
$17 / 20(85.0 \%)$
$6 / 6(100 \%)$
$39 / 43(90.7 \%)$

$31 / 36(86.1 \%)$

$6 / 6(100 \%)$

$39 / 43(90.7 \%)$

Data are presented as median (range) unless otherwise stated. ITT: intent-to-treat; XDR: extensively drug-resistant; FQ: fluoroquinolone; SLI: second-line injectable; TB: tuberculosis; PAS: para-aminosalicylic acid; AE: adverse event; BR: background regimen; QTcF: QT interval corrected for heart rate using Fridericia's method; AST: aspartate aminotransferase; ALT: alanine aminotransferase. \#: second-line drugs are all anti-TB drugs excluding rifampicin, isoniazid, pyrazinamide, streptomycin and ethambutol. ": discontinued bedaquiline due to toxic nephropathy and depression considered not related to bedaquiline by the investigator. The BR was permanently withdrawn due to depression and temporarily withdrawn due to toxic nephropathy. Depression and toxic nephropathy were considered possibly and very likely to be related to the BR, respectively, by the investigator. ${ }^{+}$: 96 -week post-bedaquiline+BR treatment phase during which patients received BR alone. ${ }^{\S}$ : pulmonary haemorrhage. ${ }^{f}$ : acute myocardial infarction. ${ }^{\# \#}$ : none was considered at least possibly related to bedaquiline by the investigator. ${ }^{\text {१ी }}$ : each of the other AEs at least possibly related to bedaquiline occurred in only one patient: hypercreatininaemia, hyperuricaemia, haematuria, leukocyturia, proteinuria, dermatitis and hypotension. ${ }^{++}$: asthma, pulmonary haemorrhage, appendicitis, pulmonary TB, psychotic disorder or cervix carcinoma stage $0 .{ }^{\S}$ : events were graded according to the Division of Microbiology and Infectious Diseases Adult Toxicity Table [15].

Of 61 screened patients, 57 were recruited and received bedaquiline plus BR (ITT population) at three sites in Russia $(n=54)$ and one site in Lithuania $(n=3)$. All patients were Caucasian and HIV negative, with a median (range) age of 28 (18-61) years (table 1); 57.9\% were female. Most patients (93\%) had previously used second-line TB drugs. Pre-treatment, 47.4\% had pre-XDR-TB (24.6\% FQ resistant and 22.8\% SLI resistant) and 52.6\% had XDR-TB.

The most frequently used TB drugs in the BR (>40\%) during the 120 -week study were FQs $(100 \%$; levofloxacin $82.5 \%$ (47 out of 57) and moxifloxacin 57.9\% (33 out of 57), with some patients switching from levofloxacin to moxifloxacin or vice versa), para-aminosalicylic acid (PAS) $(89.5 \%$, 51 out of 57 ), pyrazinamide $(87.8 \%, 50$ out of 57$)$, capreomycin $(84.2 \%, 48$ out of 57$)$, linezolid $(66.7 \%$, 38 out of 57$)$, terizidone $(61.4 \%, 35$ out of 57$)$ and cycloserine $(54.4 \%, 31$ out of 57). Prothionamide, ethambutol, amikacin sulfate, kanamycin, gatifloxacin, ofloxacin and amoxicillin/clavulanate were used by $<40 \%$ of patients.

By 120 weeks, 43 patients had completed the study (75.4\%) and 14 discontinued (24.6\%) (table 1). During 120 weeks, most AEs were grade 1 or 2 in severity and $10.5 \%$ were considered by the investigator to be related to bedaquiline. Observed grade 3-4 (26.3\%) and serious AEs (14\%) were not considered to be related to bedaquiline. The most frequent AEs, regardless of cause or severity, were increased aspartate aminotransferase (AST) and alanine aminotransaminase (ALT), and eosinophilia (table 1). Acknowledging the small study size and limitations of comparing across studies, no new clinically relevant safety findings were noted, and the pattern and severity of AEs was generally similar to that reported in the phase $2 b$ bedaquiline studies $[4,5]$. While elevated AST and ALT, and eosinophilia occurred more frequently than in the phase $2 \mathrm{~b}$ studies $[4,5]$, grade 3 ALT/AST increases were of similar incidences and no grade 4 increases were reported. None of the eosinophilia AEs or grade 3 increases in ALT or AST were considered by the investigator to be related to bedaquiline, and none led to bedaquiline discontinuation. Furthermore, most patients with ALT/AST increases were using PAS, which may be associated with hepatotoxicity and drug-induced hepatitis [16], and two of these patients had a medical history of hepatitis C.

None of the three deaths were considered to be bedaquiline or BR related by the investigator (table 1), or associated with a QT interval corrected for heart rate using Fridericia's method (QTcF) >450 ms. Two deaths occurred during the post-investigational phase (441-442 days after last bedaquiline intake); both were due to pulmonary haemorrhage and considered to be TB related by the investigator. Neither patient had culture converted (table 1). The third death occurred after week 120 (411 days after bedaquiline) and was due to an acute myocardial infarction (table 1). Overall mortality was 5\%, compared with $7 \%$ in C209 [5], 12\% in C208 stage 2 [4] and 15\% in a large meta-analysis of patients with MDR-TB [17]. 
Bedaquiline was associated with a small QTcF prolongation at week 24 (mean change from baseline $+1.4 \mathrm{~ms}$ ) that gradually declined after bedaquiline treatment ended. AEs related to QTcF prolongation considered possibly related to bedaquiline by the investigator were reported in only two patients, starting 15 and 94 days, respectively, after bedaquiline treatment. Neither event was grade 3 or 4 , or considered serious. Both events resolved with no action required (mean change in QTcF at 24 weeks was $-15 \mathrm{~ms}$ and $+123 \mathrm{~ms}$, respectively). No events of ventricular arrhythmia or torsades de pointes were reported. None of the patients received clofazimine with bedaquiline, or had a clinically significant QTcF prolongation $>500 \mathrm{~ms}$ or other ECG abnormalities.

In the 45 patients (ITT) who had a positive baseline TB culture and available post-baseline results, sputum culture conversion at 24 weeks was $68.9 \%$ (31 out of 45 ) overall, $72.7 \%$ ( 16 out of 22 ) in pre-XDR-TB and $65.2 \%$ (15 out of 23) in XDR-TB patients. Only 16 patients had sputum culture data reported at 120 weeks; six were negative and 10 were reported as "not done". Table 1 shows the analysis censoring for assessments that were not done and "unknown". The efficacy results support observations from the phase $2 \mathrm{~b}$ and real-world bedaquiline studies $[4,5,8-10]$.

A strength of the trial was its design, which reflected routine clinical practice and included a high proportion of difficult-to-treat patients. Most patients had previously received second-line TB drugs $(93 \%$ versus $86 \%$ in C209 [5]) and $>50 \%$ had XDR-TB (versus 16\% in C209). However, the study did not have strictly defined data collection goals, so a large amount of microbiological data were missing, especially towards the end of treatment, as is expected during MDR-TB therapy in the real-world setting. Aside from the small study size, other limitations were the lack of a comparator arm or inclusion of HIV-positive patients.

In conclusion, bedaquiline added to a BR was generally well tolerated and efficacious over 120 weeks, as measured by traditional endpoints in patients infected with pre-XDR-TB and XDR-TB. These findings support the inclusion of bedaquiline in an individualised BR for the treatment of MDR-TB, including $\mathrm{XDR}-\mathrm{TB}$, across a broad spectrum of patients.

Irina Vasilyeva ${ }^{1}$, Andrei Mariandyshev ${ }^{2}$, Boris Kazennyy ${ }^{3}$, Edita Davidavičiene ${ }^{4}$, Nacer Lounis ${ }^{5}$ and Sofia Keim ${ }^{6}$, on behalf of the TMC207TBC3001 study group

${ }^{1}$ National Medical Research Center of Phthisiopulmonology and Infectious Diseases, Moscow, Russia. ${ }^{2}$ Dept of Tuberculosis, Northern State Medical University, Arkhangelsk, Russia. ${ }^{3}$ Orel Oblast Tuberculosis Dispensary, Orel, Russia. ${ }^{4}$ National Tuberculosis and Infectious Diseases University Hospital, Vilnius, Lithuania. ${ }^{5}$ Janssen Pharmaceutica NV, Beerse, Belgium. ${ }^{6}$ Janssen-Cilag, Porto Salvo, Portugal.

Correspondence: Irina Vasilyeva, National Medical Research Center of Phthisiopulmonology and Infectious Diseases, Moscow, Russia. E-mail: vasil39@list.ru

Received: June 262018 | Accepted after revision: April 052019

Acknowledgements: We would like to express gratitude to the patients and their families for their participation and support during the study. We would also like to thank the study centre staff and public health authorities for their support. We are grateful to all the Janssen study personnel, as well as Nyasha Bakare and other Janssen staff members for their review of this letter, and Chris Liu, previously a contractor with Johnson \& Johnson, who was involved in the data analysis. Medical writing support was provided by Ian Woolveridge of Zoetic Science, an Ashfield company, Macclesfield, UK; this support was funded by Janssen.

This study is registered at www.clinicaltrials.gov with identifier number NCT01464762. The data sharing policy of Janssen Pharmaceutical Companies of Johnson \& Johnson is available at https://www.janssen.com/clinical-trials/ transparency. As noted on this site, requests for access to the study data can be submitted through Yale Open Data Access (YODA) Project site at http://yoda.yale.edu.

Author contributions: I. Vasilyeva, A. Mariandyshev, B. Kazennyy and E. Davidavičienè all participated in recruiting significant numbers of patients to the trial and reported data for those patients. N. Lounis and S. Keim had significant involvement in the study conception or design, and/or contributed to acquisition, analysis or interpretation of data. All authors were involved in the development of this letter and interpretation of data, have read and approved the final version, and have met the criteria for authorship as established by the International Committee of Medical Journal Editors.

Conflict of interest: I. Vasilyeva has nothing to disclose. A. Mariandyshev has nothing to disclose. B. Kazennyy has nothing to disclose. E. Davidavičiene has nothing to disclose. N. Lounis is an employee of Janssen Pharmaceutical Companies of Johnson \& Johnson. S. Keim reports is an employee of and holds shares in Janssen Pharmaceutical Companies of Johnson \& Johnson.

Support statement: This study was sponsored by Janssen. The study sponsor was involved in the design and conduct of the trial, and in the collection and analysis of the data. The corresponding author had full access to the clinical trial report and final responsibility to submit the manuscript for publication. Funding information for this article has been deposited with the Crossref Funder Registry. 


\section{References}

World Health Organization. Global Tuberculosis Report 2018 (WHO/CDS/TB/2018.20). Geneva, WHO, 2018.

2 Migliori GB, Sotgiu G, Gandhi NR, et al. Drug resistance beyond extensively drug-resistant tuberculosis: individual patient data meta-analysis. Eur Respir J 2013; 42: 169-179.

3 Pietersen E, Ignatius E, Streicher EM, et al. Long-term outcomes of patients with extensively drug-resistant tuberculosis in South Africa: a cohort study. Lancet 2014; 383: 1230-1239.

4 Diacon AH, Pym A, Grobusch MP, et al. Multidrug-resistant tuberculosis and culture conversion with bedaquiline. $N$ Engl J Med 2014; 371: 723-732.

5 Pym AS, Diacon AH, Tang SJ, et al. Bedaquiline in the treatment of multidrug- and extensively drug-resistant tuberculosis. Eur Respir J 2016; 47: 564-574.

6 Guglielmetti L, Hewison C, Avaliani Z, et al. Examples of bedaquiline introduction for the management of multidrug-resistant tuberculosis in five countries. Int J Tuberc Lung Dis 2017; 21: 167-174.

7 Udwadia ZF, Ganatra S, Mullerpattan JB. Compassionate use of bedaquiline in highly drug-resistant tuberculosis patients in Mumbai, India. Eur Respir J 2017; 49: 1601699.

8 Borisov SE, Dheda K, Enwerem M, et al. Effectiveness and safety of bedaquiline-containing regimens in the treatment of MDR- and XDR-TB: a multicentre study. Eur Respir J 2017; 49: 1700387.

9 Olayanju O, Limberis J, Esmail A, et al. Long-term bedaquiline-related treatment outcomes in patients with extensively drug-resistant tuberculosis from South Africa. Eur Respir J 2018; 51: 1800544.

10 Guglielmetti L, Jaspard M, Le Dû D, et al. Long-term outcome and safety of prolonged bedaquiline treatment for multidrug-resistant tuberculosis. Eur Respir J 2017; 49: 1601799.

11 Pontali E, D'Ambrosio L, Centis R, et al. Multidrug-resistant tuberculosis and beyond: an updated analysis of the current evidence on bedaquiline. Eur Respir J 2017; 49: 1700146.

12 Mbuagbaw L. Review of available evidence on the use of bedaquiline for the treatment of multidrug-resistant tuberculosis: data analysis report. www.who.int/tb/publications/2017/Appendix_GDGReport_Bedaquiline.pdf. Date last updated: March 8, 2017.

13 Pontali E, Sotgiu G, Tiberi S, et al. Cardiac safety of bedaquiline: a systematic and critical analysis of the evidence. Eur Respir J 2017; 50: 1701462.

14 Schnippel K, Ndjeka N, Maartens G, et al. Effect of bedaquiline on mortality in South African patients with drug-resistant tuberculosis: a retrospective cohort study. Lancet Respir Med 2018; 6: 699-706.

15 Division of Microbiology and Infectious Diseases. Adult Toxicity Table. Bethesda, US National Institutes of Health, 2007.

16 GRANUPAS (para-aminosalicylic acid) granules summary of product characteristics. Lucane Pharma, Paris, France, April 2014. www.ema.europa.eu/docs/en_GB/document_library/EPAR_-_Product_Information/human/ 002709/WC500166371.pdf. Date last accessed: June 21, 2019.

17 Ahuja SD, Ashkin $\mathrm{D}$, Avendano $\mathrm{M}$, et al. Multidrug resistant pulmonary tuberculosis treatment regimens and patient outcomes: an individual patient data meta-analysis of 9,153 patients. PLoS Med 2012; 9: e1001300. 